\title{
The Comparison of Multi-Slice CT-Scan and Laparotomy Findings in Blunt Splenic and Hepatic Trauma
}

\author{
Hamidreza Haghighatkhah, ${ }^{1}$ Hesameddin Hoseini Tavassol, $2,{ }^{*}$ Reyhane Kazemi, ${ }^{3}$ and Shaghayegh
}

Sadat Khabbaz ${ }^{3}$

${ }^{1}$ Shohada-e-Tajrish Hospital, Shahid Beheshti University of Medical Sciences, Tehran, Iran

${ }^{2}$ Shahid Beheshti University of Medical Sciences, Tehran, Iran

${ }^{3}$ Department of Radiology, Shahid Beheshti University of Medical Sciences, Tehran, Iran

"Corresponding author: Hesameddin Hoseini Tavassol, Shahid Beheshti University of Medical Sciences, Tehran, Iran. E-mail: Hesam.tavasol.md@gmail.com

Received 2016 December 21; Accepted 2017 February 08.

\begin{abstract}
Background: Liver and spleen are the most common injured organs in abdominal blunt trauma which can lead to life threatening bleeding. Fast and reliable evaluation of these injuries is important to manage them immediately. In this study, the level of agreement between multidetector CT-Scan and surgical findings is evaluated.

Methods: Multidetector CT-scan and explorative laparotomy of the patients with blunt abdominal trauma were assessed retrospectively. Characteristics of the injuries were categorized on the basis of the American Association for the Surgery of Trauma (AAST) grading system and the findings were analyzed using kappa test.

Results: The findings of 35 patients were analyzed. According to the kappa test, there were "Moderate" agreement between CT-Scan and surgery in evaluation of lacerations, location and AAST grade of splenic injuries, and disagreement in evaluation of splenic hematoma. In assessment of lacerations and AAST grading of hepatic injuries, there was only "Fair" agreement between CT-Scan and surgery.

Conclusions: Multidetector CT-Scan is an appropriate method for evaluating splenic and hepatic blunt trauma, even more accurate than surgery in some limited cases. However, only "Moderate" and "Fair" agreements, indicate that initial measures such as history taking and abdominal physical examination, specifically for unstable patients that they not have enough time to do CT-Scan, should be properly considered before using this method. Furthermore, in case of other organ injuries, CT-Scan can be an appropriate method for prioritizing other damages to splenic and hepatic injuries or vice versa, on the basis of intensity, characteristics and the grade of injury.
\end{abstract}

This is an abstract presented in the 33rd Iranian congress of radiology (ICR) and the 15th congress of Iranian radiographic science association (IRSA) 